\title{
Studies of the molecular mechanism of caspase-8 activation by solution NMR
}

\author{
N Keller ${ }^{1}$, MG Grütter ${ }^{1}$ and 0 Zerbe ${ }^{\star, 2}$
}

\begin{abstract}
Caspases are the key players of apoptosis and inflammation. They are present in the cells as latent precursors, procaspases, and are activated upon an apoptotic or inflammatory stimulus. The activation mechanism of caspases has been studied extensively by biochemical and biophysical methods. Additional structural information on active caspases with a variety of different inhibitors bound at the active site is available. In this study, we investigated the cleavage mechanism of caspase- 8 from its zymogen to active caspase-8 by solution NMR and by biochemical methods. The intermolecular cleavage reaction using the catalytically inactive C285A procaspase-8 mutant is triggered by adding caspase-8 and followed by ${ }^{15} \mathrm{~N},{ }^{1} \mathrm{H}-\mathrm{NMR}$ spectroscopy. The spectrum that initially resembles the one of procaspase-8 gradually over time changes to that of caspase-8, and disappearing peaks display exponential decaying intensities. Removal of either one of the cleavage recognition motifs in the linker, or phosphorylation at Tyr380, is shown to reduce the rate of the cleavage reaction. The data suggest that dimerization repositions the linker to become suitable for intermolecular processing by the associated protomer. Furthermore, analysis of inhibitor binding to the active caspase-8 reveals an induced-fit mechanism for substrate binding.

Cell Death and Differentiation (2010) 17, 710-718; doi:10.1038/cdd.2009.155; published online 23 October 2009
\end{abstract}

Caspases are cysteine-dependent enzymes with specificity for aspartic acid in the cleavage recognition motif of their substrates and are involved in apoptosis and inflammation. ${ }^{1-4}$ In apoptosis, initiator caspases are the most apical enzymes, which receive the apoptotic signal and activate downstream executioner caspases. Initially, caspases are produced in the cells as inactive zymogens, the procaspases. ${ }^{1-3,5}$ Inflammatory and apoptotic initiator procaspases are monomeric and contain an $\mathrm{N}$-terminal prodomain that facilitates their recruitment to macromolecular activation platforms, such as the death-inducing signaling complex (DISC), ${ }^{6,7}$ the apoptosome, ${ }^{8,9}$ the PIDDosome $e^{10,11}$ or the inflammasome. ${ }^{12}$ Through binding to these complexes their apparent concentration is increased and dimerization is induced. Dimerization then triggers the activation of initiator caspases as proposed by the induced-proximity model. ${ }^{13,14}$ Although forced into mutual proximity at the activation complexes, autoproteolytic cleavage of the inter-subunit linker occurs. A second cleavage event between the prodomain and the catalytic domain releases the active caspase from the activation complex. ${ }^{15}$ For executioner caspases cleavage of the inter-subunit linker constitutes the major event for activation, whereas for initiator caspases it is dimerization. ${ }^{16}$

Fundamental insight into the mechanism of caspase activation has been derived from crystal structures, with most of the structures of active caspases determined in the presence of inhibitors. ${ }^{17}$ Additional crystal structures of inflammatory caspase- 1 and executioner caspase- 7 in the absence of inhibitors report on structural changes upon inhibitor binding, suggesting an induced-fit mechanism of substrate binding. ${ }^{18,19}$ Furthermore, a few zymogen structures are available, for example for Drosophila melanogaster Dronc, ${ }^{20}$ for executioner procaspase- $7,{ }^{18}$ for inflammatory procaspase- $1^{21}$ and for procaspase $-8{ }^{22}$ The latter was solved by solution NMR and for the first time revealed the position of the inter-subunit linker in a monomeric zymogen.

Caspase-8 is one of the best-studied initiator caspases. The recently determined solution structure of procaspase- 8 and the available crystal structures of caspase- 8 in the presence of various inhibitors provide important information to decipher the activation mechanism of caspase-8..$^{22-25}$ Additional mutational studies dissected the interplay between dimerization and proteolytic cleavage. ${ }^{13-15,22,26-28}$ Although dimerization is required and by itself sufficient for caspase-8 activity, cleavage of the inter-subunit linker at two different recognition motifs is important for dimer stabilization and is, thus, essential for caspase-8 activity. ${ }^{22}$ Although the importance of dimerization and cleavage is established, experiments that describe the transition from the inactive zymogen to the activated and to the inhibitor-bound caspase- 8 are not available presently. Furthermore, to our knowledge a structure of caspase-8 in the absence of an inhibitor has not yet been published. In this work we interrogate the activation mechanism from monomeric, unprocessed zymogen to

\footnotetext{
${ }^{1}$ Department of Biochemistry, University of Zurich, Switzerland and ${ }^{2}$ Institute of Organic Chemistry, University of Zurich, Switzerland

*Corresponding author: O Zerbe, Institute of Organic Chemistry, University of Zurich, Zurich $\mathrm{CH}-8057$, Switzerland.

Tel: + 4144 6354263; Fax: + 4144 6356882; E-mail: oliver.zerbe@oci.uzh.ch

Keywords: caspase-8; procaspase-8; activation; dimerization; cleavage; NMR

Abbreviations: EDTA, ethylenediaminetetraacetic acid; LDAO, lauryldimethylamine-oxide; GdHCl, guanidine hydrochloride; DTT, dithiothreitol; HSQC, heteronuclear single quantum coherence; TROSY, transverse relaxation optimized spectroscopy; TCEP, tris(2-carboxyethyl)phosphine; ATP, adenosintriphosphate; AMC, 7-amino-4methylcoumarin; cmk, chloro-methylketone

Received 29.5.09; revised 16.9.09; accepted 17.9.09; Edited by G Salvesen; published online 23.10.09
} 
processed, dimeric caspase in the absence of inhibitors using solution NMR as the primary tool. We follow the cleavage reaction in the NMR tube by monitoring procaspase-8 processing using ${ }^{15} \mathrm{~N},{ }^{1} \mathrm{H}$ correlation NMR spectroscopy. In addition, we determine the extent to which efficiency of caspase- 8 processing is influenced by the dimerization propensity, by phosphorylation at Tyr380 and by limited proteolysis of the inter-subunit linker. Finally, NMR data are used to evaluate how the structure of the free caspase- 8 differs from the inhibitor-bound form.

\section{Results}

Processing of procaspase-8 into active caspase-8. To characterize the events leading to the activation of caspase-8 on a molecular level, we followed the cleavage process from procaspase- 8 to active caspase- 8 by NMR. ${ }^{15} \mathrm{~N}$-labelled procaspase was mixed with catalytic amounts $(1: 10000)$ of unlabelled caspase-8. Processing of procaspase- 8 by caspase-8 was monitored by recording $\left[{ }^{15} \mathrm{~N},{ }^{1} \mathrm{H}\right]$-TROSY spectra. As only procaspase-8 is labeled, initially the spectrum resembled the one of procaspase-8, for which an assignment was previously obtained ${ }^{22}$ and gradually over time changed into the spectrum corresponding to that of caspase-8. At the beginning of the experiment the narrower lines of procaspase-8 allowed the recording of spectra in $1 \mathrm{~h}$ intervals. During biochemical processing a decrease of the signal intensity in the spectra occurred for most resonances reflecting the formation of dimeric caspase- 8 accompanied by slight precipitation. Significant line broadening is observed for methyl resonances (Figure 1b) correlating well with increased processing as independently monitored by SDSPAGE (Figure 1a).

Although most of the peaks remained unchanged, a significant number of peaks decreased in intensity and new peaks appeared (Supplementary Figure S1). A few very strong peaks appeared in the random coil region. As these signals were absent in the spectra of separately produced caspase-8, in which the inter-subunit fragment was removed during purification, they most likely correspond to the fragment excised during cleavage.

Comparing peak integrals over time we observed an exponential decay of all peaks (Figure 1c). After approximately $30 \mathrm{~h}$ the peaks in the $\left[{ }^{15} \mathrm{~N},{ }^{1} \mathrm{H}\right]$-TROSY spectra contain about $30 \%$ of their original intensity. An example is depicted in Figure 1c, were signals from the C-terminus, which is identical in procaspase-8 and caspase-8, are presented. In contrast, signal intensity for all regions affected by processing is below $5 \%$ of their initial values. Unfortunately, the time resolution is insufficient to allow distinguishing whether events occur simultaneously or in sequential order.

Caspase-8 undergoes structural changes during processing and during inhibitor binding. Processing of procaspase- 8 into caspase- 8 is accompanied by large
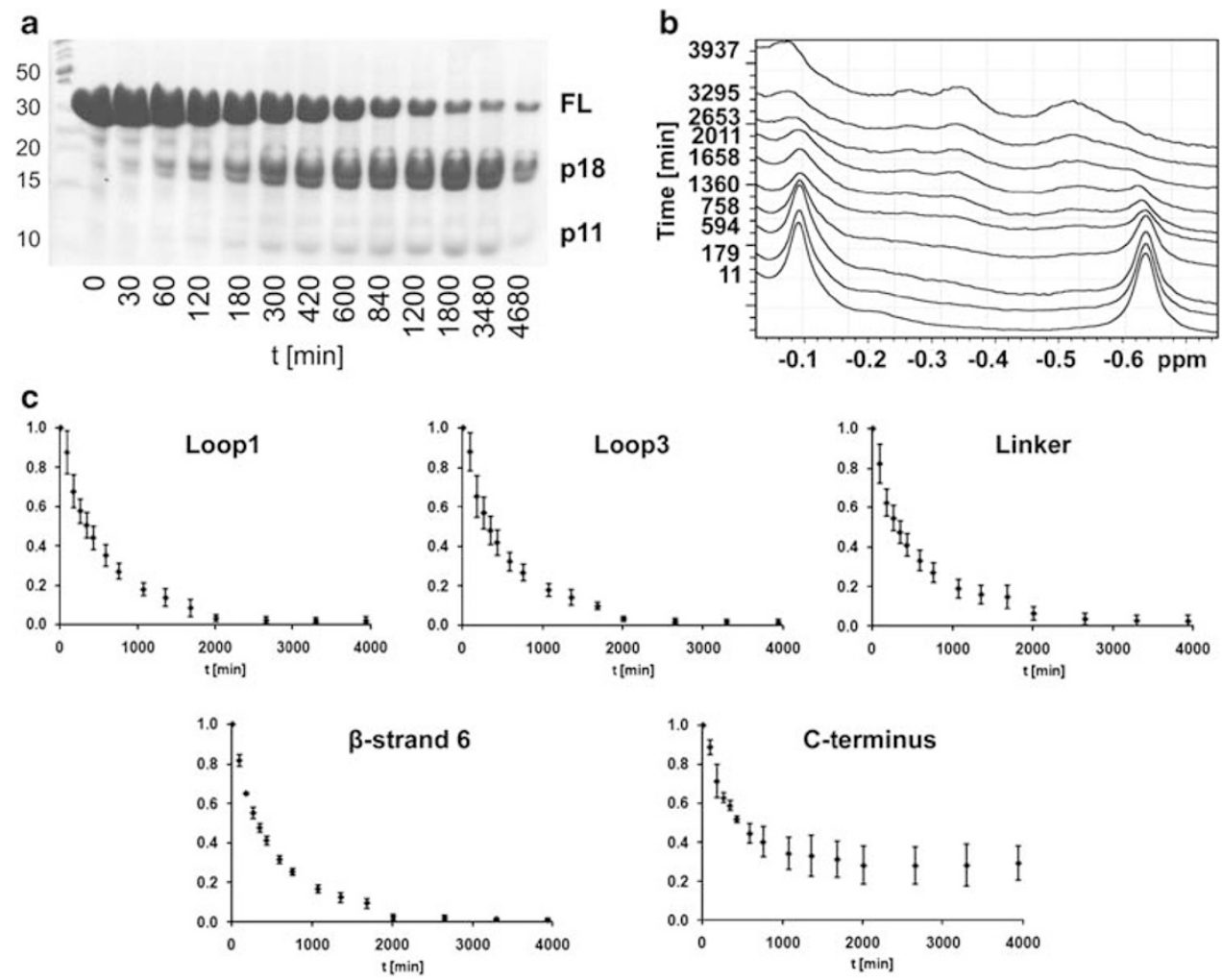

Figure 1 Processing of procaspase-8 into caspase-8. Labeled procaspase-8 was incubated in a 1:10000 ratio with unlabelled caspase-8. Cleavage was monitored simultaneously by SDS-PAGE and NMR. (a) SDS-PAGE with procaspase-8 samples obtained at indicated time points during the cleavage process. (b) Regions of 1D proton spectra displaying the methyl region recorded at indicated time points. (c) Time course of signal decrease in $\left[{ }^{15} \mathrm{~N},{ }^{1} \mathrm{H}\right]-\mathrm{TROSY}$ spectra. Peak intensities present averages for all residues from the segment indicated on the plots. Error bars reflect the standard deviation from the mean 
spectral changes that are difficult to interpret. Furthermore, during the long measurement time the precipitation of protein resulted in a decrease in protein concentration, thus leading to a reduction in signal intensity. Therefore, we decided to characterize the product of the cleavage reaction in its purified form. Accordingly, caspase-8 was prepared from the active wild-type procaspase-8 in ${ }^{15} \mathrm{~N},{ }^{2} \mathrm{H}$-labelled form. The similarity of $\left[{ }^{15} \mathrm{~N},{ }^{1} \mathrm{H}\right]-\mathrm{HSQC}$ and $\left[{ }^{15} \mathrm{~N},{ }^{1} \mathrm{H}\right]-\mathrm{TROSY}$ spectra recorded on the cleavage-incompetent C285A procaspase- 8 mutant and caspase- 8 indicates that both proteins are structurally highly similar (Figure 2a). Only signals corresponding to the loops and the linker as well as from residues of the $\beta$-strands 5 and 6 , which in procaspase8 are in close proximity to the intact linker, were significantly shifted in position. In addition, $\beta$-strand 6 forms the dimerization interface in active caspase-8, and therefore residues from these strands are expected to experience chemical shift changes.

During processing, the oligomerization state of the enzyme changes (Table 1). At the approximate concentration used in the NMR experiments procaspase-8 is purely monomeric, whereas caspase-8 which is known to exist in a concentration-dependent monomer/dimer equilibrium, ${ }^{22,27}$ is present as a dimer at $81 \%$. The increasing population of dimeric states during caspase- 8 activation is also apparent from the line widths of methyl signals in proton spectra (Figure $2 \mathrm{~b}$ ). In addition, the peaks are shifted in position, thereby indicating that they belong to residues undergoing structural changes or become involved in forming intermolecular contacts. a

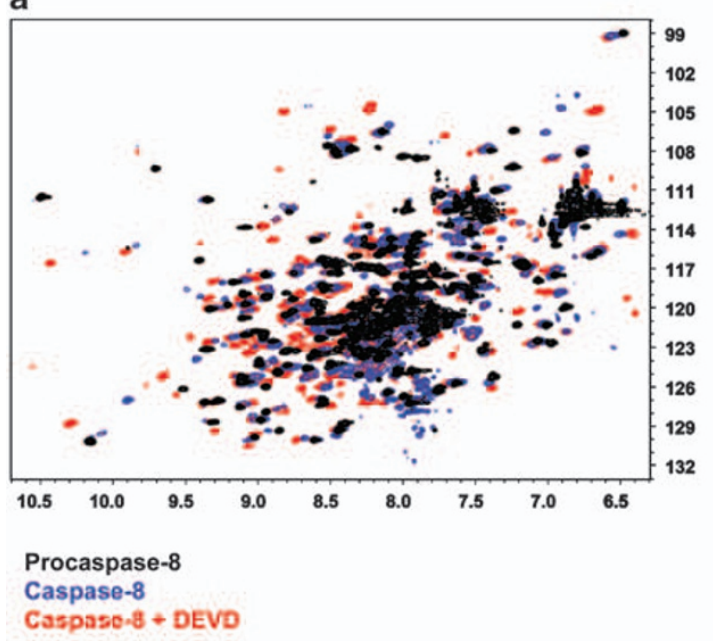

b
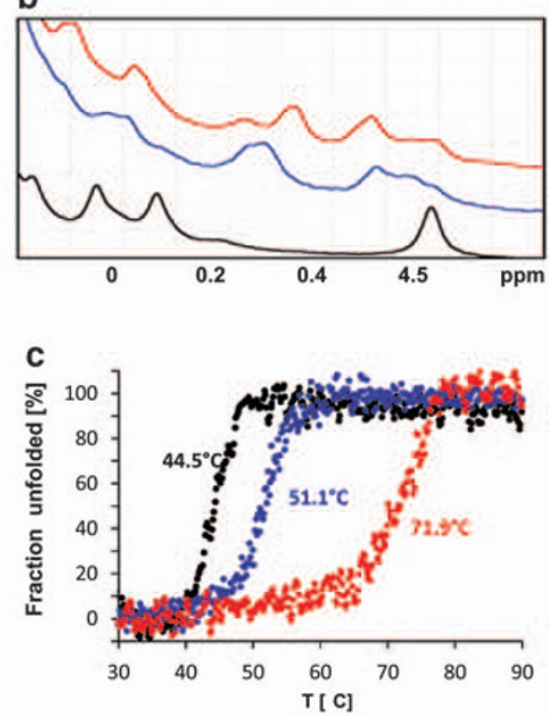

Figure 2 Comparison of procaspase-8 (black) with caspase-8 in the absence (blue) and presence (red) of the tetrapeptidic DEVD inhibitor. (a) Overlay of a $\left[{ }^{15} \mathrm{~N},{ }^{1} \mathrm{H}\right]-\mathrm{HSQC}$ spectrum with $\left[{ }^{15} \mathrm{~N},{ }^{1} \mathrm{H}\right]$-TROSY spectra of caspase-8 in the absence or presence of the Ac-DEVD-cmk inhibitor. Differences in peak positions indicate changes in the environment of the corresponding amide moieties due to conformational changes or contacts to the inhibitor. (b) Section of the high-field region of a 1D proton spectrum displaying methyl signals. Line widths are correlated with protein size such that increasing molecular weight during dimerization results in broader lines. Furthermore, differences in peak positions were observed as also seen in (a). (c) Thermal denaturation of procaspase-8 and caspase-8 before and after inhibitor binding followed by CD spectroscopy. CD signals recorded at $222 \mathrm{~nm}$ were used to calculate the fraction of folded protein. The melting temperatures for the individual proteins are indicated in the plot

Table 1 Data derived from analytical ultracentrifugation of constructs used for the NMR and the cleavage experiments

\begin{tabular}{lcc}
\hline Mutant & Sedimentation coefficient [S $_{\text {exp }}$ ] & Monomer/dimer (\%) \\
\hline Constructs used in NMR experiments $(800 \mu M)$ & $2.5 \pm 0.031 /-$ & $100 /-$ \\
Procaspase-8 (C285A) & $2.3 \pm 0.022 / 3.9 \pm 0.035$ & $19 / 81$ \\
Caspase-8 & $2.2 \pm 0.021 / 3.5 \pm 0.034$ & $11 / 89$ \\
Caspase-8+DEVD & & \\
Constructs used in cleavage experiments $(250 \mu M)$ & $2.5 \pm 0.019 / 3.7 \pm 0.029$ & $33 / 67(29 / 71)^{\mathrm{a}}$ \\
Procaspase-8 (C285A) & $2.4 \pm 0.019 / 3.9 \pm 0.031$ & $44 / 56$ \\
D374A (C285A) & $2.7 \pm 0.018 / 3.8 \pm 0.025$ & $67 / 33$ \\
D384A (C285A) & $2.5 \pm 0.019 /-$ & $100 /-$ \\
F468A & & \\
\end{tabular}

Samples were measured at concentrations of 800 and $250 \mu \mathrm{M}$ for NMR and cleavage experiments, respectively. Constructs used for the cleavage experiments harbor the active-site mutation $\mathrm{C} 285 \mathrm{~A}$ and were processed by caspase-8 addition at a 1:1000 ratio. ${ }^{\text {a }}$ The value in brackets was obtained previously at the same concentration for active caspase-8, which underwent autoproteolytic processing. ${ }^{22}$ 
Structural information is available for procaspase- 8 and inhibitor-bound caspase-8, but not for the inhibitorfree enzyme. The above-described data allows comparing caspase-8 in its zymogen and in the inhibitor-free state. To assess how the free enzyme differs from the published inhibitor-bound crystal structure, we added the irreversible Ac-DEVD-cmk inhibitor and monitored changes by chemical shift mapping. It is interesting to note that significant changes in peak positions occur. Analysis of the crystal structure of DEVD bound to caspase- 8 reveals that about 21 amino acids are either directly involved in inhibitor binding or are in close vicinity. Surprisingly, comparison of the $\left[{ }^{15} \mathrm{~N},{ }^{1} \mathrm{H}\right]-\mathrm{TROSY}$ spectra of caspase- 8 in presence and absence of the inhibitor reveals that more than 70 peaks are shifted, suggesting further structural changes to take place upon inhibitor binding. Considering that the majority of the secondary structure elements are identical, it is likely that these peaks belong to the active site forming loops or be located at the dimerization interface. However, the population of dimer in the presence versus absence of inhibitor as determined by analytical ultracentrifugation is 81 and $89 \%$ (Table 1), respectively, and hence the difference is too small to explain the changes observed in the spectra. Moreover, a number of new peaks appear with rather strong intensity that must belong to residues in more flexible regions such as the active-site forming loops.

To access the influence of activation and inhibitor binding on the overall protein stability the melting points of the proteins were determined by CD spectroscopy (Figure 2c). Dimerization and cleavage of the zymogen results in improved protein stability characterized by a 6.6-degree increase in the melting temperature for caspase- 8 compared with procaspase-8. This effect highlights the contribution of intermolecular contacts from residues of the cleaved linker and at the terminal $\beta$-strand to protein stability and provides a rationale for the driving force for cleavage and dimerization. An even more pronounced stabilizing effect was observed upon covalent inhibitor binding to caspase-8, which elevates the melting temperature by approximately 20 degrees.

Determinants of the processing rate of procaspase-8. In the following we analyze the extent to which the rate of processing of procaspase- 8 by the addition of catalytic amounts of the active enzyme is influenced by the propensity for dimerization, by phosphorylation of procaspase- 8 and by insufficient cleavage of the inter-subunit linker. Therefore, we mutated either one of the Asp cleavage motifs in the linker, introduced a mutation at the dimerization interface or phosphorylated procaspase-8 in vitro (Figure 3). To prevent self-activation the constructs harbor an additional C285A mutation. To all the mutants active caspase- 8 was added in catalytic amounts $(1: 1000)$, the reaction was stopped at indicated time points, and the rate of processing was analyzed by SDS-PAGE and densitometry. Over time the intensity of the bands corresponding to full-length protein decreased and new bands for $\mathrm{p} 18 / \mathrm{p} 19$ and $\mathrm{p} 11 / \mathrm{p} 12$ subunits became visible. Integration of the bands corresponding to full-length protein revealed a double-exponential decay (Figure 4a). Although the procaspase-8 mutants are rapidly processed in the beginning of the experiment cleavage rates

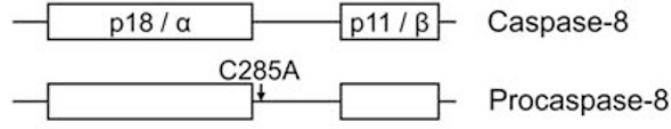

Cleavage Mutants

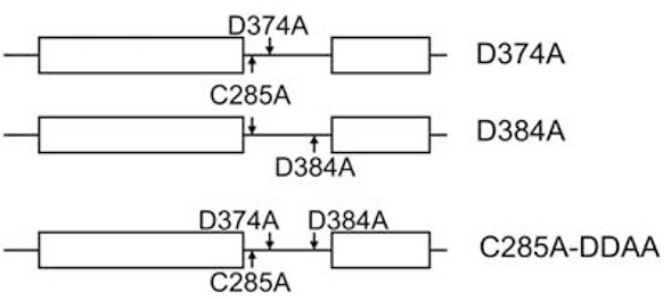

Dimerization mutant

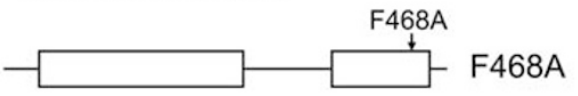

Phosphorylated procaspase

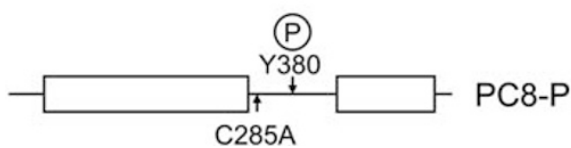

Figure 3 Overview of caspase-8 mutants used in this study indicating positions of individual mutation sites

are reduced later on. We believed this to be mainly due to a decrease in enzymatic activity of caspase- 8 over time, and therefore determined the activity of caspase-8 using the fluorogenic Ac-DEVD-AMC substrate. After $3000 \mathrm{~min}$ caspase- 8 retains $1 \%$ of its original activity (Figure $4 b$ ). The same experiment was carried out by further addition of 1000 equivalents of the procaspase- 8 mutant C285A-DDAA that cannot be cleaved because of the double mutation D374A D384A (DDAA) and also cannot process other substrates. This mutant does not compete with the AcDEVD-AMC substrate for the active site of caspase- 8 in contrast to procaspase-8. Surprisingly, addition of C285ADDAA resulted in increased caspase-8 activity (Figure 4c) and retarded caspase-8 deactivation (Figure 4b), thereby indicating that it is able to interact with caspase-8, most likely via heterodimerization. Furthermore, analytical ultracentrifugation revealed that the affinity for dimer formation in cleaved procaspase-8 is only slightly lower than for caspase-8 (Table 1).

Despite the time-dependent deactivation of caspase-8, a comparison of the cleavage efficiencies for the various substrates can still be made because all cleavage reactions have been carried out under identical conditions, and hence deactivation of caspase-8, and therefore remaining active caspase- 8 is comparable in all samples at identical time points. It is interesting to note that all the analyzed mutants are less efficient in processing when compared with procaspase8. Although initial processing rates are similar within the range of the experimental error, pronounced differences are observed after approximately $5-10 \mathrm{~h}$. In case of the Asp 

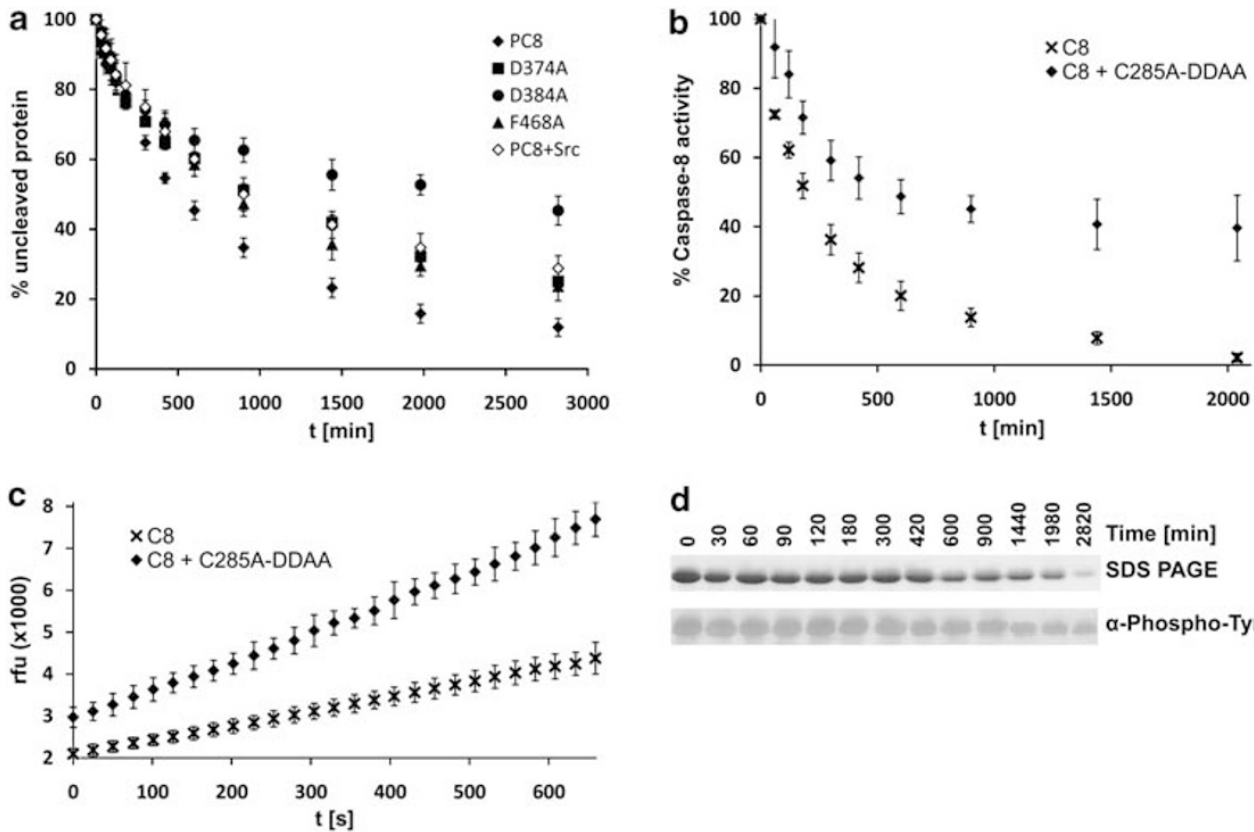

d
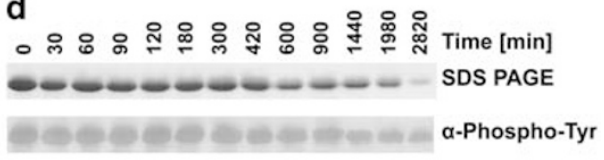

Figure 4 Processing of various procaspase-8 mutants by caspase-8. (a) Time course of processing for various mutants (see panel) of procaspase-8 displaying the percentage of unprocessed protein at indicated time points. Processing was determined by SDS-PAGE and densitomety on bands corresponding to uncleaved enzyme. (b) Time-dependent deactivation of caspase-8 in the absence and presence of the inactive uncleavable C285A-DDAA mutant monitored by conversion of the fluorogenic Ac-DEVD-AMC substrate. (c) Influence of inactive uncleavable procaspase-8 C285A-DDAA on caspase-8 activity. (d) SDS-PAGE and corresponding western blot of phosphorylated procaspase-8 following addition of caspase-8

mutants the processing rate is more strongly affected for D384A. Analytical ultracentrifugation data of the cleavage products of both mutants reveals that the affinity for dimerzation is impaired compared with cleaved procaspase-8, and that this reduction is larger for D384A than for D374A. Moreover, F468A shows a significant decrease in the processing rate as compared with procaspase- 8 underlining the important role of dimerization for activation.

Previous studies by Cursi et al. ${ }^{29}$ revealed a negative effect on Fas-induced apoptosis after phosphorylation of caspase-8 by Src kinase. Src kinase phosphorylates caspase-8 specifically at Tyr380, which is located between the two cleavage recognition motifs within the linker and is excised during processing. In this study, we phosphorylated caspase-8 in vitro with Src kinase. Phosphorylation of caspase-8 was detected by western blot using a specific phospho-tyrosine antibody (Figure 4d). The rate of conversion for phosphorylated procaspase-8 is slower than for its unphosphorylated counterpart (Figure 4a). It is interesting to note that the band observed on SDS-PAGE decreases faster in intensity than the band on the western blot, indicating that the unphosphorylated enzyme is preferably cleaved (Figure 4d). Phosphorylation at Tyr380 can reduce the rate of processing, and thus caspase- 8 activation and apoptosis by either sterically blocking access to the cleavage sites or by altering the conformation of the linker.

\section{Discussion}

The activation of inflammatory and apoptotic initiator procaspases proceeds through dimerization, which is stabilized by subsequent proteolytic processing. And although our understanding of the underlying molecular processes has greatly advanced with the development of important concepts like the induced-proximity model ${ }^{13,14}$, the exact molecular details are still not fully understood. Only a few procaspase structures are available such as for Drosophila melanogaster Dronc, ${ }^{20}$ procaspase- $7,{ }^{18}$ procaspase- $1^{21}$ and procaspase- $8 .{ }^{22}$ Much more structural information is available on active caspases in the presence of bound inhibitors, whereas at present no structural information is available for inhibitor-free apoptotic initiator caspases. In contrast structures describing the dimers of caspase- 1 and -7 have been published in both the inhibitor free and inhibitor-bound states. ${ }^{18,19}$

In this work, we investigated the cleavage of procaspase-8 and subsequent binding of substrates applying chemical shift mapping and biochemical methods. In the conducted experiments trace amounts of active caspase- 8 triggered conversion of procaspase- 8 mutants that are deficient of autoprocessing. Previous studies on procaspase- 8 and caspase- 8 stated that caspase- 8 has no specificity for its own zymogen. ${ }^{15}$ The experiments presented in this work, however, clearly demonstrate processing of the zymogen after the addition of caspase- 8 indicating that caspase- 8 does have specificity for procaspase-8.

The cleavage of procaspase- 8 results in an apparent increase in molecular weight, and large structural differences in the loops and at the dimerization interface. In principle, cleavage can be accomplished in three different ways: (i) by an intramolecular process, (ii) by an intermolecular process in which the participating Asp and Cys residues are part of different protomers within the same dimer (intradimeric) 
or (iii) by an intermolecular process involving a proximal caspase dimer (interdimeric). All constructs that were tested for cleavage contained the active-site mutation of C285A and are incapable of self-processing and therefore require intermolecular cleavage. The observation that an inactive, cleavage-incompetent C285A-DDAA mutant increases the activity of caspase-8, thereby suggesting that heterodimerization between procaspase- 8 and caspase- 8 does occur. Considering the vast excess of the procapase- 8 mutants over active capase-8 (1000: 1 in the biochemical and 10000: 1 in the NMR experiments) caspase- 8 is more likely to associate with procaspase-8 than with itself, which supports the intradimeric cleavage hypothesis. In addition, the concentration of caspase- 8 was in the nano-molar range, wherein caspase- 8 homodimer formation ( $\mathrm{Kd}$ of approximately $50 \mu \mathrm{M}^{27}$ ) is unlikely, whereas our assay concentration of procaspase-8 $(250 \mu \mathrm{M})$ was well above this threshold. Although the affinity of caspase-8 for procaspase-8 may be lower than for caspase-8, formation of the heterodimer could result in immediate processing. Finally, intra-dimeric attack by Cys285 is expected to be favored kinetically. Our in vitro experiments support this view, as they reveal a correlation between dimerization and cleavage of the substrate. Processing is slowed down in the dimerization-incompetent F468A mutant. Although $\mathrm{F} 468 \mathrm{~A}$ is incompetent for homodimerization because cross-strand intermolecular interactions of Phe468 with Pro466 have been removed, due to the pseudo-symmetry in the heterodimer one of the two Pro-Phe contacts remain. We expect the $\mathrm{Kd}$ for formation of the heterodimer to be lower than the corresponding value for formation of the homodimer in caspase-8, and this is reflected in the reduced processing rate. The connection between rate of processing and affinity for dimerization for the cleaved enzyme might also explain the reduced processing rates for the cleavage mutants $D 374 A$ and $\mathrm{D} 384 \mathrm{~A}$. Both mutants display a reduction in dimerization affinity, which is more pronounced in the slower processing D384A mutant.

If such intra-dimeric attack takes place, the linker must then undergo a dramatic conformational change in order to bring Asp374 and Asp384 into the proximity of catalytic Cys285 from the other protomer. One plausible scenario is that the relocation of the linker is triggered by dimerization that pushes loop 4 and possibly loop 5 towards that active site, thus displacing the linker segment from that region. We noticed that resonances from loops 4 and 5 were completely absent in the ${ }^{15} \mathrm{~N},{ }^{1} \mathrm{H}$-correlation spectra of procaspase-8, indicating that these two loops interconvert between at least two different states, and hence intrinsically sample a larger conformational space. 22

The proposed conformational change is very large, but precedence for such a linker topology is found in the crystal structure of procaspase-1. Although procaspase-1 is monomeric in solution, it was crystallized as a dimer with the linker located in a cleft at the dimer interface and the cleavage motif for the first processing site positioned close to the active site of the second protomer (Figure $5 c,{ }^{21}$ ). Furthermore, the linker in procaspase- 1 is similarly positioned as the cleaved linker of active caspase-1 and -8 (Figure 5a). Loops 3 and 4 (corresponding to loops 4 and 5 in procaspase-8) are slightly shifted towards the active site supporting their role in linker displacement. Thus, the structure of procaspase- 1 displays all structural elements that are required for the proposed mode of cleavage for procapase- 8 , and we have taken this as a model of how the structure of procaspase- 8 could change during dimerization.

Previous studies state that dimerization is the prime event in initiator caspase activation that triggers structural rearrangements of the active site forming loops leading to formation of the active site. ${ }^{14,22,27,28,30,31}$ Our chemical shift mapping experiment using the DEVD inhibitor indicates that
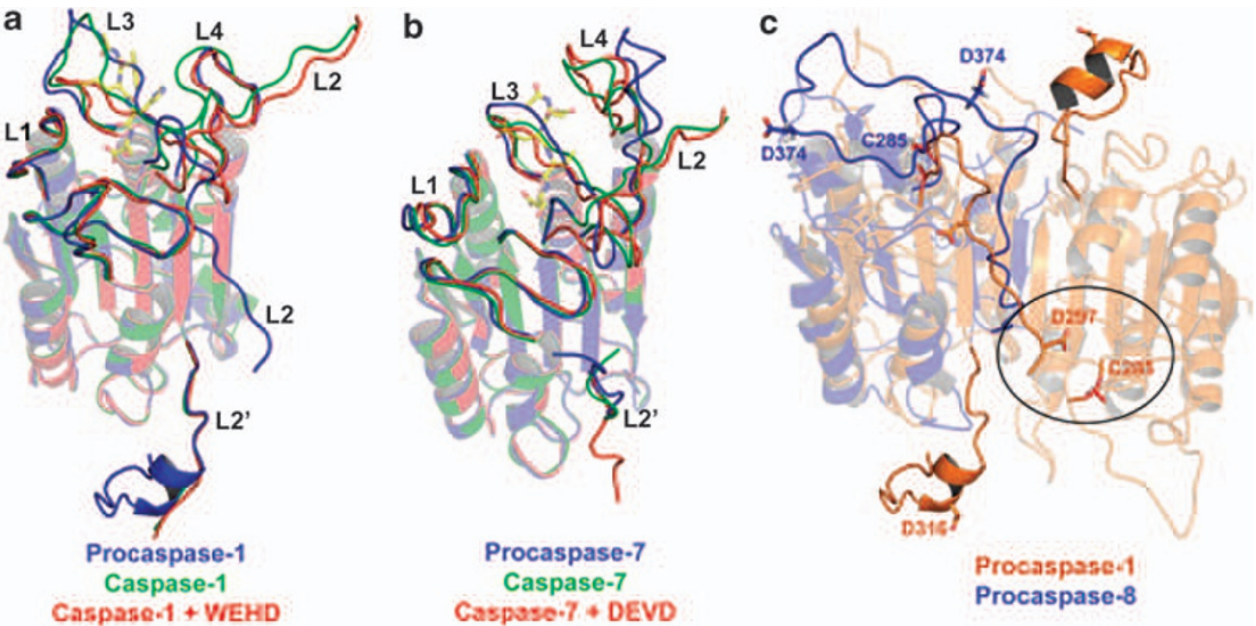

Figure 5 Comparison of different caspase activation states. (a) Superposition of procaspase-1 (blue, pdb code $3 \mathrm{E} 4 \mathrm{C}$ ) and caspase-1 in the absence (green, pdb code 1SC1) and presence (red, pdb code 1/BC) of a tetrapeptidic WEHD inhibitor (yellow). The active site forming loops are labeled and indicated in bright colors. The tetrapeptidic inhibitor is presented as sticks. (b) Superposition of procaspase-7 (pdb code 1K88) and caspase-7 in the absence (pdb code 1K86) and presence (pdb code 1F1J) of a tetrapeptidic DEVD inhibitor. The color coding and labeling is the same as described in (a). (c) Comparison of the linker orientation in dimeric procaspase-1 (brown) and monomeric procaspase-8 (blue, pdb code $2 \mathrm{~K} 7 \mathrm{Z}$ ). The active site Cys and the Asp residues in the cleavage motifs are presented as sticks. All figures were prepared using $\mathrm{PyMo}^{37}$ 


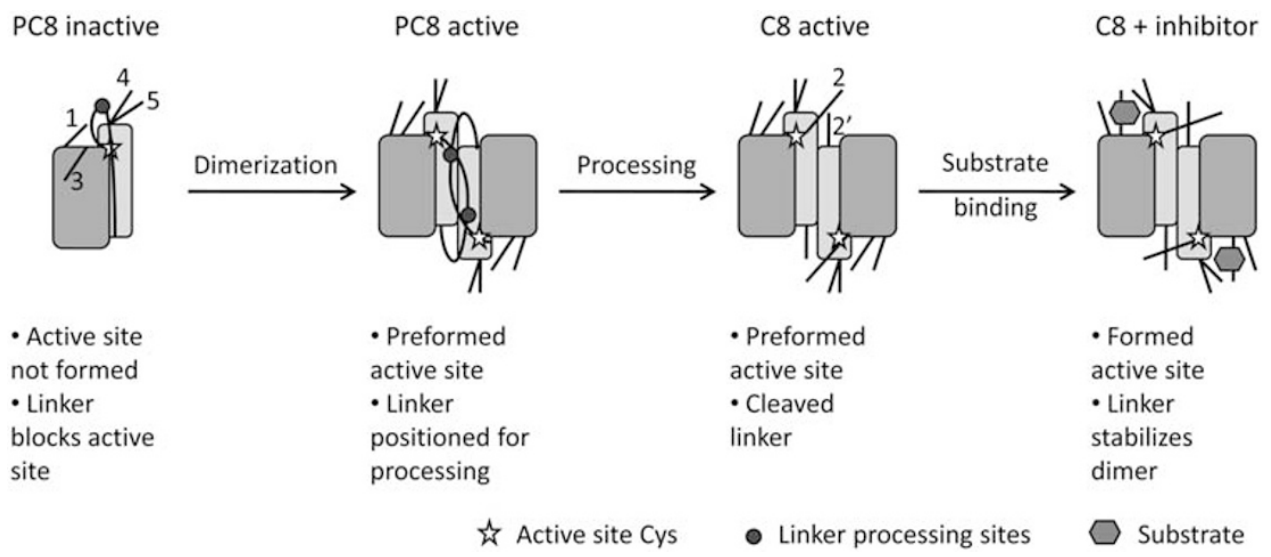

Figure 6 Schematic drawing of the caspase-8 activation mechanism. The large and small subunits are indicated by large and small boxes, respectively. The active site forming loops and the linker are depicted as lines and labeled according to the nomenclature used in Keller et al. ${ }^{22}$

dimerization is not sufficient to form a proper active site, and that additional significant changes occur upon inhibitor binding. This statement is supported by similar conclusions drawn from the comparison of crystal structures of caspase-1 and caspase-7 in the presence or absence of inhibitors and their corresponding zymogens. ${ }^{18,19}$ In both enzymes structural differences are observed in the active site forming loops 3 and 4 and in the linker fragments with the inhibitor-free form being a structural intermediate between the zymogen and the inhibitor-bound species (Figure 5b). Moreover, loops 3 and 4 are arranged in a way that the substrate-binding pocket is not properly formed in the absence of inhibitors. Furthermore, the cleaved linker fragments $\mathrm{L} 2$ and $\mathrm{L}^{\prime}$ ' of the associated protomers interact with each other in all inhibitor-bound crystal structures while they are separated in inhibitor-free caspase-7. ${ }^{18}$ Mutants of caspase-8, in which the L2 loop is truncated, experience a significant shift towards the monomeric state for caspase-8 suggesting their importance for dimer stabilization. ${ }^{22}$ Structural differences of the L2 loop between inhibitor-free and inhibitor-bound caspase-8 could explain the higher dimer affinity upon inhibitor binding, a common phenomenon for initiator caspases. ${ }^{27}$

Previous experiments on caspase-7 revealed an increased overall protein stability upon inhibitor binding by 17.9 degrees, ${ }^{32}$ which is in the same range as observed for caspase-8 and in case of dimeric caspase-7 must be independent of dimerization. We therefore speculate that the increased overall stability of caspase-8 upon addition of the inhibitor is due to rigidification of the loops around the active site and possibly may also involve loop 4 and 5 , which in procaspase-8 undergo conformational exchange. ${ }^{22}$

Structures for inhibitor-free caspases have also been determined for caspase- $3^{33,34}$ and $-9 .^{31}$ Caspase- 9 belongs to the initiator caspases, and the proenzyme is monomeric, indicating that the mechanism of action might be highly similar to the one of caspase-8. The crystal structure of dimeric caspase- 9 , however, revealed that only one active site within the dimer is occupied by the inhibitor, whereas the unbound protomer reveals a different conformation with characteristics of the proform. ${ }^{31}$ Obviously, the inhibitor-bound species of caspase- 9 is different than for caspase-8, and therefore a comparison of the mechanisms is difficult on the basis of the presently available data. Rearrangements upon inhibitor binding in the executioner caspase-3 are limited to side chains of three hydrophobic residues lining the S2 pocket and no loop stabilization was observed ${ }^{33,34}$ in contrast to the more extensive changes in capase-8. To what extent the unbound structure of caspase-3 differs from the zymogen cannot be assessed, as no structural information on the proform is available.

These observations together with our data suggest that substrate binding to the active-site pocket during caspase activation probably generally occurs via an induced-fit mechanism. Whether the changes in caspase-8 involve larger conformational changes of the loops, as observed for caspase-1 and -7 , or minor side chain rearrangements, as seen in caspase-3, cannot be distinguished based on our experiments. However, the data confirm that dimerization is the prime event during caspase- 8 activation and allow expanding the current model of initiator caspase activation (Figure 6). We propose that dimerization helps relocating the cleavage motifs within the linker to become accessible for intermolecular cleavage and initiates pre-building of the active site, which finally forms upon substrate binding.

Caspase- 8 activation at the DISC is a very complex issue that is difficult to address in vitro and certainly requires additional biochemical experiments under conditions that better mimic the DISC environment. The model for caspase-8 activation proposed in this work provides a working hypothesis that may help guiding the design of such experiments.

\section{Materials and Methods}

Protein design, expression and purification. The DNA sequence coding for human caspase-8 amino acids 217-479 was cloned into a PQE50 vector (Qiagen, Valencia, CA, USA). Amino-acid substitutions were generated by Quikchange (Stratagene, La Jolla, CA, USA) mutagenesis. The plasmids were transformed into M15 (pREP4) cells (Qiagen), and the protein was expressed at $37^{\circ} \mathrm{C}$ overnight either in LB (unlabeled protein) or in M9 $\left({ }^{15} \mathrm{~N}\right.$-labeled protein) medium. M9 minimal media was supplemented with ${ }^{15} \mathrm{NHCl}_{4}$ as the sole nitrogen source. Deuteration was achieved by growing cells in $\mathrm{D}_{2} \mathrm{O}$. 
The purification protocol was adapted from published procedures ${ }^{25}$ and was described previously. ${ }^{22}$ Cells were harvested, resuspended in PBS buffer and lyzed by french press. The soluble protein fraction was removed by centrifugation, inclusion bodies washed in $20 \mathrm{mM}$ Tris pH 8.0, $1 \mathrm{mM}$ EDTA, 0.1\% LDAO and solubilized in $6.5 \mathrm{mM} \mathrm{GdHCl}, 20 \mathrm{mM}$ Tris $\mathrm{pH} 8.0,2 \mathrm{mM}$ EDTA, $100 \mathrm{mM}$ DTT. The solubilized inclusion bodies were added to an equal volume of acetic acid and the sample dialyzed against $50 \%$ acetic acid. Subsequently the solution was suspended rapidly in nine volumes $\mathrm{H}_{2} \mathrm{O}$ and immediately diluted in ninefold excess of $1 \mathrm{M}$ Tris pH 8.0, $5 \mathrm{mM}$ DTT. Refolding was carried out overnight at room temperature and aggregates were removed by centrifugation. Final purification was achieved by size exclusion chromatography on a Superdex 200 column (Amersham, Piscataway, NJ, USA) using $20 \mathrm{mM}$ Tris, $\mathrm{pH} 8.0,100 \mathrm{mM} \mathrm{NaCl}$, $10 \%$ sucrose, $0.5 \mathrm{mM}$ TCEP. In this buffer the protein can be stored at $-80^{\circ} \mathrm{C}$. Light scattering was used to confirm monodispersity of the refolded purified sample.

NMR experiments. Nuclear magnetic resonance samples were rebuffered into $20 \mathrm{mM}$ deuterated Tris $\mathrm{pH} 8.0,100 \mathrm{mM} \mathrm{KCl}, 10 \mathrm{mM}$ DTT. Procaspase-8 that was used to monitor the time course of cleavage and active caspase- 8 was both ${ }^{2} \mathrm{H},{ }^{15} \mathrm{~N}$-labelled. The irreversible tetrapeptidic AC-DEVD-cmk inhibitor was added in fivefold excess to ${ }^{2} \mathrm{H},{ }^{15} \mathrm{~N}$-labelled active caspase-8. All NMR experiments were recorded at $305 \mathrm{~K}$ on a Bruker Avance $700 \mathrm{MHz}$ spectrometer, equipped with a triple-resonance cryoprobe. $\left[{ }^{15} \mathrm{~N},{ }^{1} \mathrm{H}\right]-\mathrm{HSQC}$ and $\left[{ }^{15} \mathrm{~N},{ }^{1} \mathrm{H}\right]$-TROSY experiments were recorded during cleavage for monomeric proteins and of dimeric caspase-8, respectively. The spectra were processed in Topspin2.1 (Bruker, Karlsruhe Germany) and transferred to CARA for spectral analysis. ${ }^{35}$ Complete processing at both cleavage sites was confirmed by MALDI mass spectroscopy.

Analytical ultracentrifugation. Analytical ultracentrifugation was carried out with an Optima XL-1 centrifuge (Beckman Coulter, Fullerton, CA, USA) and a TI50 rotor using epoxy centerpieces $(12 \mathrm{~mm})$ with sapphire windows. Samples were run at $20^{\circ} \mathrm{C}$ and 42000 r.p.m. Absorption was measured between 280 and $315 \mathrm{~nm}$, depending on protein concentration. Results were analyzed using Sedfit. ${ }^{36}$

CD spectroscopy. Thermal denaturation was performed on a Jasco J-715 spectropolarimeter equipped with a temperature control unit. The concentrations of the samples were $100 \mu \mathrm{M}$ using a quartz cuvette with a $1 \mathrm{~mm}$ path length. Data points were taken at $222 \mathrm{~nm}$ while increasing the temperature by $1^{\circ} \mathrm{C} / \mathrm{min}$ from 5 to $95^{\circ} \mathrm{C}$. The band width was set to $1 \mathrm{~nm}$ and the response time to $4 \mathrm{~s}$.

In vitro cleavage experiments. All proteins were dialyzed against phosphorylation buffer ( $50 \mathrm{mM}$ Tris pH 8.0, $150 \mathrm{mM} \mathrm{NaCl}, 0.5 \mathrm{mM}$ TCEP, $20 \mathrm{mM}$ $\mathrm{MgCl}_{2}, 12.5 \mathrm{mM} \mathrm{MnCl}_{2}$ and $12.5 \mathrm{mM} \beta$-glycerolphosphate). Phosphorylation of procaspase-8 was accomplished by incubation with Src kinase (SignalChem, Richmond, CA, USA) with 25-fold excess of procaspase and 5 -fold excess of ATP at $37^{\circ} \mathrm{C}$ for $3 \mathrm{~h}$. Small amounts of precipitated protein were removed by centrifugation. The final sample volume for monitoring the cleavage reaction was $200 \mu \mathrm{l}$ containing $250 \mu \mathrm{M}$ procaspase- 8 mutants. The reaction was started with the addition of $250 \mathrm{nM}$ caspase-8. Small aliquots were removed at indicated time points, immediately suspended in hot $5 \times$ SDS loading buffer and incubated for $5 \mathrm{~min}$ at $95^{\circ} \mathrm{C}$. The cleavage educts and products were separated by SDS-PAGE. The gels were scanned in a Fuji LAS 3000 image reader (FujiFilm, Tokyo, Japan) and the bands corresponding to full-length caspase-8 analyzed by densitometry using the AIDA image analyzer software. The obtained values were normalized by setting the concentration of full-length protein at the beginning of the reaction to $100 \%$ and by appropriate scaling of all subsequent data. Measurements were carried out in triplicate and the S.Ds. were calculated. Initially we tried to fit the kinetic data against single exponentially decaying functions, but discovered that data could not be fit well because of inactivation of caspase- 8 . Inactivation of caspase-8 in the absence and presence of PC8 DDAA was determined by addition of the AC-DEVD-AMC substrate at a final concentration of $100 \mu \mathrm{M}$. The concentrations of the proteins and the buffer were the same as used in the cleavage reactions described above. Measurements were carried out in triplicate in black 96-well Nuncion microtiter plates using the Magellan6 software on a GENios plate reader (Tecan, Crailsheim, Germany). AMC release was monitored by its fluorescence at $465 \mathrm{~nm}$ following excitation at $360 \mathrm{~nm}$.

\section{Conflict of interest}

The authors declare no conflict of interest.
Acknowledgements. This work was supported by the Swiss National Science Foundation (grant 31-1022181 to MG Grütter) and the European Commission (CAMP Project LSHG-2006-018830). We thank Professor A Baici for valuable discussions of the cleavage data. Nadine Keller is a member of the $\mathrm{PhD}$ Program in Molecular Life Sciences, University of Zurich and ETH Zurich, Switzerland.

1. Salvesen GS, Riedl SJ. Caspase mechanisms. Adv Exp Med Biol 2008; 615: 13-23.

2. Denault JB, Salvesen GS. Apoptotic caspase activation and activity. Methods Mol Biol 2008; 414: 191-220.

3. Rupinder SK, Gurpreet AK, Manjeet S. Cell suicide and caspases. Vascul Pharmacol 2007; 46: 383-393.

4. Kumar S. Caspase function in programmed cell death. Cell Death Differ 2007; 14: 32-43.

5. Taylor RC, Cullen SP, Martin SJ. Apoptosis: controlled demolition at the cellular level. Nat Rev Mol Cell Biol 2008; 9: 231-241.

6. Weber $\mathrm{CH}$, Vincenz $\mathrm{C}$. A docking model of key components of the DISC complex: death domain superfamily interactions redefined. FEBS Let 2001; 492: 171-176.

7. Roy A, Hong JH, Lee JH, Lee YT, Lee BJ, Kim KS. In vitro activation of procaspase-8 by forming the cytoplasmic component of the death-inducing signaling complex (CDISC). Mol Cells 2008; 26: 165-170.

8. Yu X, Acehan D, Menetret JF, Booth CR, Ludtke SJ, Riedl SJ et al. A structure of the human apoptosome at $12.8 \AA$ resolution provides insights into this cell death platform. Structure 2005; 13: 1725-1735.

9. Riedl SJ, Salvesen GS. The apoptosome: signalling platform of cell death. Nat Rev $\mathrm{Mol}$ Cell Biol 2007; 8: 405-413.

10. Tinel A, Tschopp J. The PIDDosome, a protein complex implicated in activation of caspase2 in response to genotoxic stress. Science 2004; 304: 843-846.

11. Park HH, Logette E, Raunser S, Cuenin S, Walz T, Tschopp J et al. Death domain assembly mechanism revealed by crystal structure of the oligomeric PIDDosome core complex. Cell 2007; 128: 533-546.

12. Petrilli V, Dostert C, Muruve DA, Tschopp J. The inflammasome: a danger sensing complex triggering innate immunity. Curr Opin Immunol 2007; 19: 615-622.

13. Muzio M, Stockwell BR, Stennicke HR, Salvesen GS, Dixit VM. An induced proximity model for caspase-8 activation. J Biol Chem 1998; 273: 2926-2930.

14. Boatright KM, Renatus M, Scott FL, Sperandio S, Shin H, Pedersen IM et al. A unified model for apical caspase activation. Mol Cell 2003; 11: 529-541.

15. Chang DW, Xing Z, Capacio VL, Peter ME, Yang X. Interdimer processing mechanism of procaspase-8 activation. EMBO J 2003; 22: 4132-4142.

16. Liu $\mathrm{H}$, Chang DW, Yang X. Interdimer processing and linearity of procaspase-3 activation. A unifying mechanism for the activation of initiator and effector caspases. $\mathrm{J}$ Biol Chem 2005; 280: 11578-11582.

17. Chowdhury I, Tharakan B, Bhat GK. Caspases - an update. Comp Biochem Physiol 2008; 151: $10-27$

18. Chai J, Wu Q, Shiozaki E, Srinivasula SM, Alnemri ES, Shi Y. Crystal structure of a procaspase-7 zymogen: mechanisms of activation and substrate binding. Cell 2001; 107: 399-407.

19. Romanowski MJ, Scheer JM, O'Brien T, McDowell RS. Crystal structures of a ligand-free and malonate-bound human caspase-1: implications for the mechanism of substrate binding. Structure 2004; 12: 1361-1371.

20. Yan N, Huh JR, Schirf V, Demeler B, Hay BA, Shi Y. Structure and activation mechanism of the Drosophila initiator caspase Dronc. J Biol Chem 2006; 281: 8667-8674.

21. Elliott JM, Rouge L, Wiesmann C, Scheer JM. Crystal structure of procaspase-1 zymogen domain reveals insight into inflammatory caspase autoactivation. J Biol Chem 2009; 284 : $6546-6553$.

22. Keller N, Mares J, Zerbe O, Grütter MG. Structural and biochemical studies on procaspase8: new insights on initiator caspase activation. Structure 2009; 17: 438-448.

23. Blanchard H, Donepudi M, Tschopp M, Kodandapani L, Wu JC, Grütter MG. Caspase-8 specificity probed at subsite S4: crystal structure of the caspase-8-Z-DEVD-cho complex. $\mathrm{J}$ Mol Biol 2000; 302: 9-16.

24. Blanchard H, Kodandapani L, Mittl PR, Marco SD, Krebs JF, Wu JC et al. The threedimensional structure of caspase-8: an initiator enzyme in apoptosis. Structure Fold Des 1999; 7: 1125-1133.

25. Watt W, Koeplinger KA, Mildner AM, Heinrikson RL, Tomasselli AG, Watenpaugh KD. The atomic-resolution structure of human caspase-8, a key activator of apoptosis. Structure Fold Des 1999; 7: 1135-1143.

26. Chen M, Orozco A, Spencer DM, Wang J. Activation of initiator caspases through a stable dimeric intermediate. J Biol Chem 2002; 277: 50761-50767.

27. Donepudi M, Sweeney AM, Briand C, Grütter MG. Insights into the regulatory mechanism for caspase-8 activation. Mol Cell 2003; 11: 543-549.

28. Pop C, Fitzgerald P, Green DR, Salvesen GS. Role of proteolysis in caspase-8 activation and stabilization. Biochemistry 2007; 46: 4398-4407.

29. Cursi S, Rufini A, Stagni V, Condo I, Matafora V, Bachi A et al. Src kinase phosphorylates caspase-8 on Tyr380: a novel mechanism of apoptosis suppression. EMBO J 2006; 25 1895-1905. 
30. Pop C, Timmer J, Sperandio S, Salvesen GS. The apoptosome activates caspase-9 by dimerization. Mol Cell 2006; 22: 269-275.

31. Renatus M, Stennicke HR, Scott FL, Liddington RC, Salvesen GS. Dimer formation drives the activation of the cell death protease caspase 9. Proc Natl Acad Sci USA 2001; 98: 14250-14255.

32. Witkowski WA, Hardy JA. L2' loop is critical for caspase-7 active site formation. Prot Sci 2009; 18: 1459-1468

33. Ni CZ, Li C, Wu JC, Spada AP, Ely KR. Conformational restrictions in the active site of unliganded human caspase-3. J Mol Recognit 2003; 16: 121-124.
34. Agniswamy J, Fang B, Weber IT. Conformational similarity in the activation of caspase-3 and -7 revealed by the unliganded and inhibited structures of caspase-7. Apoptosis 2009; 14: $1135-1144$.

35. Keller R. The Computer Aided Resonance Assignment. CANTINA Verlag, 2004

36. Schuck P, Perugini MA, Gonzales NR, Howlett GJ, Schubert D. Size-distribution analysis of proteins by analytical ultracentrifugation: strategies and application to model systems. Biophys J 2002; 82: 1096-1111.

37. DeLano WL. The PyMOL Molecular Graphics System. DeLano Scientific LLC: San Carlos, CA, USA.

Supplementary Information accompanies the paper on Cell Death and Differentiation website (http://www.nature.com/cdd) 